\title{
社区主客观特征对社区满意度的影响机理 以上海市郊区为例
}

\author{
申 悦 ${ }^{1,2}$, 傅行行 ${ }^{3}$ \\ (1. 华东师范大学中国现代城市研究中心, 上海 $200062 ； 2$. 华东师范大学城市与区域科学学院, 上海 200062; \\ 3. 南京大学建筑与城市规划学院, 南京 210093)
}

\begin{abstract}
摘 要: 在以人为本发展理念的指导下, 城市研究与规划越来越关注城市生活空间的构建与居民生活质量的提升, 社区满意度研究的重要性不断增加。在郊区快速发展和社区生活圈构建的背景下,论文基于对上海市郊区居民活 动日志调查的一手数据, 在 Campbell模型的基础上建立理论模型, 从客观和主观相结合的视角出发, 聚焦社区客 观建成环境、社区主观感知特征和社区满意度三者之间的影响机理, 并利用结构方程模型开展实证研究。研究结 果表明: 居民的社区感知情况对社区满意度有直接性和决定性的影响, 居民对社区的健康、便利、安全和美观情况 的感知对社区满意度都存在显著的正影响; 社区的密度、可达性、设施等客观建成环境通过影响社区感知特征而间 接地影响社区满意度; 居民的社会经济属性对社区感知特征和社区满意度的影响甚微, 影响郊区居民社区满意度 的主要因素是社区特征。
\end{abstract}

关 键 词: 建成环境; 社区感知特征; 社区生活圈; 结构方程模型; 郊区; 上海

在经济社会发展全面转型的背景下,中国社会 主要矛盾发生了根本性转变, 人民对美好生活的需 求日益增加, 长期以来 “重数量, 轻质量” “见物不见 人”的城市发展观得到全面反思, 以人为本成为城 市发展的重要指导思想(宁越敏, 2012; 柴彦威等, 2014)。与此同时, 新型城镇化从传统城镇化侧重 数量规模增加转向注重内涵质量的提升, 逐渐开始 关注城市生活空间的构建与居民生活质量的提升, 对城市规划的公共性、政策性与社会性提出了新的 发展要求,而居民对重构紧凑、完整、便捷的生活空间 的诉求也日益强烈(仇保兴, 2012; 姚士谋等, 2014)。

社区作为城市生活的基本空间单元, 是人们日 常居住、休闲等活动的主要场所, 近年来社区逐渐 成为 “城市研究人本导向”下的研究热点。为了应 对社区发展所面临的趋势与挑战, 北京、上海、广州
等大城市先后提出构建 $15 \mathrm{~min}$ 社区生活圈, 并将其 作为组织城镇与乡村社区生活的基本单元, 立足 “人”的实际生活空间与需求, 统筹居民日常生活诸 要素(奚东帆等, 2017)。例如, 《上海市城市总体规 划(2017-2035 年)》提出 “以 15 分钟社区生活圈组 织紧凑复合的社区网络, 促进生活、就业、休闲相互 融合, 提升市民的幸福感”(上海市人民政府, 2016)。

社区满意度指居民对社区各个方面的总体感 受及主观评价, 是一个综合性、多层次的指标体系, 是人们微观感受的累积结果, 也是居住满意度乃至 生活满意度的重要组成部分(单菁菁, 2008; Cao et al, 2016; 袁媛等, 2018)。近年来, 随着居民生活水 平的提高, 主观幸福感和满意度的研究快速增加, 成为城市研究领域的重要议题之一(王丰龙等, 2015)。社区满意度的研究对于社区的治理与规划

收稿日期: 2018-08-03; 修订日期: 2019-01-25。

基金项目: 国家自然科学基金项目(41871166,41601159); 上海市教育发展基金会和上海市教育委员会“晨光计划”项目(17CG20); 中央高校基本科研业务费项目(2018ECNU-QKT001)。 [Foundation: National Natural Science Foundation of China, No. 41501180 and 41601159; "Chenguang Program" Supported by Shanghai Education Development Foundation and Shanghai Municipal Education Commission, No. 17CG20; Fundamental Research Funds for the Central Universities, No. 2018ECNU-QKT001. ]

第一作者简介: 申悦(1987一), 女, 上海人,副教授, 主要研究方向为城市地理学与行为地理学。E-mail: shenyue0519@163.com 引用格式: 申悦, 傅行行. 2019. 社区主客观特征对社区满意度的影响机理: 以上海市郊区为例 [J]. 地理科学进展, 38(5): 686-697. [Shen Y, Fu X X. 2019. Impact of the built environment and perceived neighborhood characteristics on residents' satisfaction: Evidence from the Shanghai suburbs. Progress in Geography, 38(5): 686-697. ] DOI: 10.18306/dlkxjz.2019.05.006 
具有借鉴意义, 有助于提升居民的社区归属感, 提 高社区品质。

中国正在经历快速的城市郊区增长,城市开发 迅速向郊区尤其是远郊地区蔓延, 郊区已然成为城 市空间的重要组成部分以及城市化的最前沿地区 (吴缚龙等, 2015; 申悦, 塔娜等, 2017)。然而, 由于 郊区各类设施的发展相对滞后, 独特的郊区化机理 又造成了中国的城市郊区产生了与西方有很大差 异的高度异质化的空间和多样化的社会群体(魏立 华等, 2006; Zhou et al, 2008; 顾朝林, 2017; 申悦等, 2018)。因此, 郊区能否发展成为真正意义上的城 市 “次中心”, 郊区社区能否满足居民多样化的需 求, 仍然面临诸多挑战, 也就需要学者们关注郊区 社区, 尤其是由于不同原因迁居至郊区、居住在不 同类型社区的居民的社区满意度情况。

本文从以人为本的城市研究和城市规划理念 出发, 聚焦上海市郊区居民的社区满意度, 研究社 区满意度的影响机理。研究将社区的客观和主观 因素相结合, 关注社区客观建成环境、居民感知的 社区环境对社区满意度的影响, 探讨面向提高居民 社区满意度的郊区社区空间优化和设施配置问题, 从而为上海“2035”社区生活圈规划提供研究依据, 以期提高居民的满意度以及生活质量。

\section{1 研究综述与理论模型}

社区满意度是生活满意度的重要组成部分, 在 很大程度上体现了居民的生活质量, 因此社区满意 度是国外社区研究和城市研究领域的热点议题之 一(Hur et al, 2008; Lovejoy et al, 2010), 主要研究内 容包括社区满意度的测量、模式和影响因素(Wang D et al, 2016; Wang F et al, 2016)。

在社区满意度的影响因素研究方面, Lovejoy 等(2010)对 1980-2010年相关实证研究中存在相关 性的变量进行了总结, 将变量分为物理环境、区位 和可达性、设施和服务、社会环境、经济环境以及个人 特征 6 大类。其中, 个人特征一般作为控制变量纳 人到模型中, 性别、年龄、受教育程度、家庭收人等个 人因素与社区满意度的相关性在不同的研究中存在 较大的差异(Baba et al, 1989; Lu, 1999; Permentier et al, 2011)。社会环境包括犯罪率、社会联系、种族混合 度等因素(Adams, 1992; Permentier et al, 2011), 其对 社区满意度的影响是社会学和心理学等领域讨论较 多的话题,一些研究发现社区的社会联系越强, 居
民的社区满意度越高(Cook, 1988; Ansari, 2002)。 经济环境包括社区房价和租金、社区住房自有率等 因素(Lu, 1999; Lovejoy et al, 2010)

地理学和城市规划等学科更关注社区的建成 环境、区位与可达性、设施与服务等客观环境因素 对社区满意度的影响。例如, 已有研究发现, 安静 的社区环境、较高的绿化率和具有吸引力的外观能 够提高社区满意度(Ansari, 2002)。社区的区位一 般通过与城市中心、工作地点的距离等指标进行测 度(Ansari, 2002)。可达性通常与设施和服务相互 联系,其中可达性指标包括公共交通可达性、商业 设施可达性、休闲健身设施可达性等,设施和服务 包括社区的车位数、所在学区的教学质量、政府机 构数量与服务质量等因素(Lee et al, 1983; Permentier et al, 2011)。这些指标都属于社区的客观特征, 在实证研究中一般采用回归分析方法考察其对社 区满意度的影响。

同时,有研究认为个体对社区的偏好有所不 同, 居住时长等客观差异也会造成主观感受的不 同,相比于社区客观特征,居民对社区特征的主观 感知及评价会对社区满意度产生更加直接的影响, 因此更关注社区的主观特征。例如, Permentier 等 (2011)研究了居民对社区人口混合度、居民交往状 况、社区安全状况、社区商业和绿化空间的评价对 其社区满意度的影响;Hur 等(2008)将居民对地方 政府服务质量、商业设施步行可达性、社区外观等 15 项社区主观评价指标作为社区满意度的解释变 量。通过相关文献分析, 可将社区主观特征归纳为 5 种类型: 设施可达性等便利特征、步行友好性等健 康特征、景观绿化等外观特征、社区治安等安全特 征、社区交往等社群特征; 已有实证研究多采用因 子分析和回归分析相结合的方法分析社区主观特 征对社区满意度的影响。

上述研究通常是对社区满意度及其影响因素 之间相关性的探讨, 理论意义存在不足, 且研究结 论较难对规划实践进行有效指导, 因此需要厘清社 区客观特征、社区主观特征和社区满意度之间的关 系, 深人挖掘社区特征与社区满意度之间的因果关 系与内在机理。有研究将社区的客观特征和主观 特征同时作为自变量放人回归模型中,分析它们对 社区满意度的影响,但这种方式不仅会导致自变量 之间存在共线性,还会使客观特征的影响被低估 (Cao et al, 2016; 袁媛等, 2018)。

Campbell模型为厘清这三者之间的关系提供了 
一个理论框架(图 1)。在 Campbell 模型中, 社区的 客观特征是社区感知和社区评价的来源, 社区感知 和社区评价是社区客观特征和社区满意度的中介 变量, 个人特征则会在整个过程中发挥作用(Campbell et al, 1976; 袁媛等, 2018)。Cao(2015)在研究社 区建成环境对生活满意度的影响时, 对 Campbell模 型进行了适当修改(图2), 研究社区客观特征、社区 感知特征、居住满意度和生活满意度之间的相互作 用机制, 发现社区客观特征对居住满意度存在直接 影响, 同时也通过社区感知特征产生间接影响。

近年来, 国内出于人居环境改善和“宜居城市” 建设的需要, 关注居住满意度及其影响因素的研究 逐渐增加。许多研究结合当前社会发展现状, 聚焦 较为突出的社会问题与现象, 例如, 有研究关注老 年人、被动迁居居民、新市民等特定群体(夏永久 等, 2013; 许丹纯等, 2014; 张景秋等, 2015; 李俊峰 等, 2017), 也有研究聚焦大型居住区、高档商品房、 衰退邻里、城中村改造安置区、小产权房小区等特 定类型社区 (赵东霞等, 2012; 邹晖等, 2014; 段雪 辉, 2016; 王娟, 2016; 冯健等, 2017), 还有研究关注 社会资本、信息通信技术等特定因素的影响(刘志 林等, 2015; 陈卉等, 2016)或居住满意度对迁居意 愿的影响(何深静等, 2014)。

而在社区满意度影响因素的研究中, 更多研究 主要关注社区的主观特征, 通过问卷调查获得居民 对社区环境的主观感知或评价以及社区满意度, 采 用回归分析等方法考察主观特征与社区满意度间 的相关性(湛东升等, 2014; 党云晓等, 2016)。关注 社区客观特征的研究相对较少, 对社区可达性、建 成环境等客观要素影响的探讨有所不足, 尤其缺乏 将主客观特征相结合而深人挖掘其与社区满意度

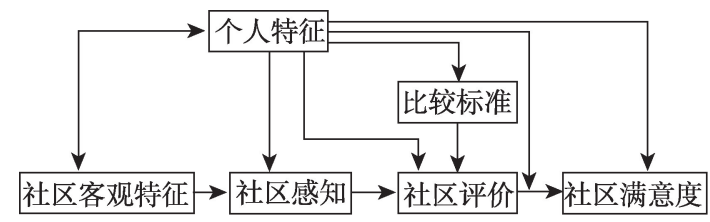

图 1 Campbell模型(Campbell et al, 1976)

Fig.1 Campbell's Model (Campbell et al, 1976)

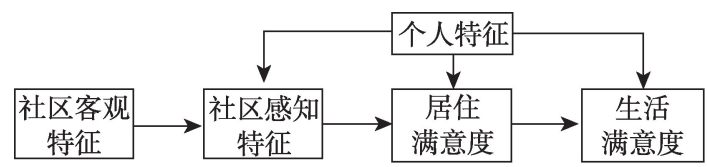

图 2 调整后的 Campbell 模型(Cao, 2015)

Fig.2 Adjusted Campbell's Model (Cao, 2015)
之间内在机理的研究。而厘清社区客观环境、社区 感知特征与社区满意度之间的相互作用机制有助 于理解如何通过改善社区环境提高居民的满意度, 为社区的规划和改造提供研究依据。

本文借鉴Campbell 模型及 Cao(2015)建立的调 整后的 Campbell模型,构建研究的理论模型。利用 结构方程模型分析社区客观特征、社区感知特征和 社区满意度之间的相互作用机制, 从客观和主观因 素相结合的视角出发, 研究中国城市郊区居民社区 满意度的影响因素, 是对社区满意度相关理论、研 究和应用的一次综合实践, 对社区满意度研究和社 区发展实践具有重要意义。

\section{2 研究数据}

\section{1 数据来源}

本文的研究数据来源于华东师范大学时空间 行为研究团队在 2017 年开展的“上海市居民日常活 动与出行调查”。调查采取多阶段抽样方法, 在上 海市选择除崇明区以外的郊区 8 个行政区,结合空 间位置、区域发展以及社区类型多样性特征, 分别 选取闵行区梅陇镇、宝山区顾村镇、嘉定区江桥镇、 松江区九亭镇、青浦区徐泾镇、奉贤区南桥镇、金山 区朱泾镇以及浦东新区的张江镇、周浦镇和南汇新 城镇为典型区域。在每个乡镇街道中结合社区类 型(商品房社区、售后公房社区、拆迁安置房社区、 社会保障房社区、城中村等)选取 3 7个社区, 在最终 选取的 58 个社区中开展随机抽样以及社区人户问 卷调查(图 3)。

问卷调查以个人为调查单元, 调查内容包括居 民的个人与家庭社会经济属性、住房情况、日常活 动与出行情况、生活中的主观感受和情感体验等内 容。调查形式为调查员半访谈式询问与手持移动 设备(平板电脑)相结合。经过 2 轮调研, 受访者总 数为 1593 人,根据问卷填写的完整性和研究需要对 数据进行校正和笁选, 最终得到有效问卷 1140 份, 问卷有效率为 $71.56 \%$ 。

\section{2 数据内容}

本文所采用的数据有 4 类, 分别是居民的社区 满意度、对社区的感知特征、社会经济属性, 以及社 区的客观特征。

居民的社区满意度通过问卷中的问题“对目前 居住的社区是否满意”进行测度,一个从“非常不满 意”(1)到“非常满意”(5)的五级量表供受访者作答, 
以此衡量居民对所居住社区满意度的综合评价。 居民对社区的感知特征反映了其对于社区的主观 评价, 结合国内外既有研究, 本文将社区感知特征

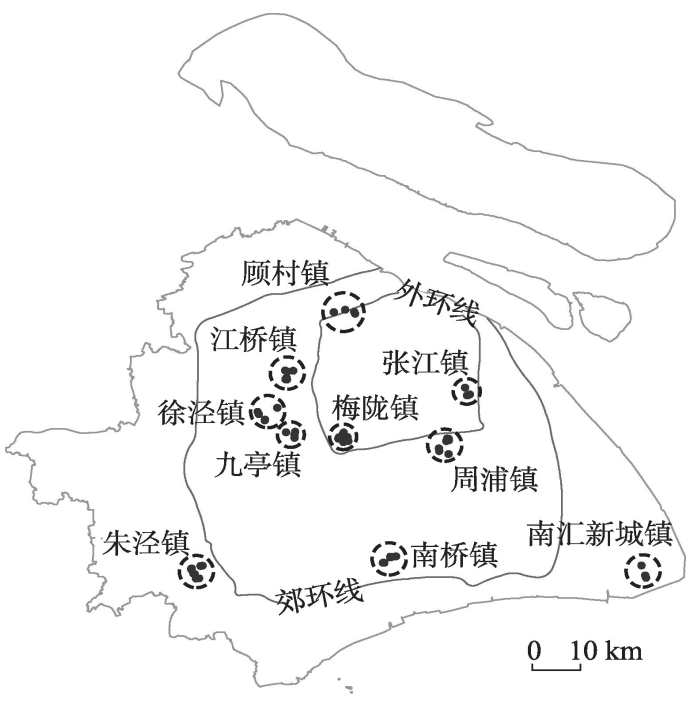

图 3 调查区域分布

Fig.3 Spatial distribution of the survey areas in the Shanghai suburbs
分为健康、便利、安全、美观和交通拥堵等方面,通过 问卷中的 5 个问题来获得: 1) 社区环境总体健康; 2) 社区环境总体便利;3) 社区环境总体安全;4) 社 区环境总体美观;5) 社区周边交通拥堵,用一个从 “非常不同意”(1)到“非常同意”(5)的五级量表来衡 量居民对社区特征的感知。居民的家庭和个人社 会经济属性来源于受访者在问卷中填写的基本 信息。

在社区的客观特征方面,将社区环境测量范围 界定为居住地附近 $1 \mathrm{~km}$ 以内, 即步行 $15 \mathrm{~min}$ 可达 的区域,对相关指标进行测度。其中,公交车站可 达性、地铁站可达性、公园广场可达性、商业密度利 用 ArcGIS 对 POI数据进行空间分析获得, 社区绿地 率、社区是否有室外游乐设施由实地调研和对各居 委会的访谈获得。此外,还将受访者的职住距离作 为客观特征加以考虑, 通过受访者的居住与就业地 的直线距离进行测度。

\section{3 样本社会经济属性}

对受访者的个人及家庭社会经济属性进行统计 (表1), 由于在调查过程中, 结合第六次人口普查结

表 1 样本社会经济属性统计

Tab.1 Sample socioeconomic characteristics

\begin{tabular}{|c|c|c|c|c|}
\hline 属性 & 类别 & 数量 & 比例 $/ \%$ & 备注 \\
\hline \multirow[t]{2}{*}{ 性别 } & 男性 & 575 & 50.4 & \\
\hline & 女性 & 565 & 49.6 & \\
\hline \multirow[t]{4}{*}{ 年龄 } & 18 30 岁 & 333 & 29.2 & \\
\hline & 30 40 岁 & 334 & 29.3 & \\
\hline & 40 50 岁 & 268 & 23.5 & \\
\hline & 50 60 岁 & 205 & 18.0 & \\
\hline \multirow[t]{2}{*}{ 户口类别 } & 上海 & 602 & 52.8 & \\
\hline & 外地 & 538 & 47.2 & \\
\hline \multirow[t]{2}{*}{ 婚姻状况 } & 已婚 & 902 & 79.1 & \\
\hline & 未婚 & 238 & 20.9 & \\
\hline \multirow[t]{6}{*}{ 就业情况 } & 全职就业 & 817 & 71.7 & 有稳定工作 \\
\hline & 半职就业 & 26 & 2.3 & 有稳定工作 \\
\hline & 临时性就业 & 15 & 1.3 & 无稳定工作 \\
\hline & 在校学生 & 48 & 4.2 & 无稳定工作 \\
\hline & 离退休在家 & 112 & 9.8 & 无稳定工作 \\
\hline & 无业 & 122 & 10.7 & 无稳定工作 \\
\hline \multirow[t]{5}{*}{ 家庭平均月收人 } & 5000 元以下 & 166 & 14.6 & \\
\hline & $5000 \sim 10000$ 元 & 418 & 36.7 & \\
\hline & $10000 \sim 20000$ 元 & 410 & 36.0 & \\
\hline & $20000 \sim 30000$ 元 & 88 & 7.7 & \\
\hline & 30000 元以上 & 58 & 5.1 & \\
\hline \multirow[t]{2}{*}{ 住房产权状况 } & 有住房产权 & 728 & 63.9 & \\
\hline & 无住房产权 & 412 & 36.1 & \\
\hline \multirow[t]{2}{*}{ 家庭是否有小汽车 } & 有小汽车 & 664 & 58.2 & \\
\hline & 无小汽车 & 476 & 41.8 & \\
\hline
\end{tabular}


果对各乡镇的各类样本比例进行了一定的控制, 因 此样本具有比较好的代表性。在 1140 个有效样本 中, 男性和女性分别占 $50.4 \%$ 和 $49.6 \%$, 男女比例平 衡; 各年龄段受访者分布较为均匀; 有上海户口的样 本略多于外地户口者; 已婚的受访者占绝大多数 (79.1\%); 约有 $80 \%$ 的样本拥有稳定的工作; 受访者 的家庭平均月收人集中在 5000 20000元之间; 有住 房产权和家中拥有小汽车的受访者比例相对较高。

\section{3 社区满意度特征}

\section{1 社区满意度的总体特征}

对样本社区满意度的总体情况进行统计发现， 选择“比较满意”的样本比例最高( $48.3 \%$ ), 其次为 “一 般”(34.9\%), 选择其他 3 个选项的比例远低于这 2 项， 其中选择 “非常不满意” 的居民比例最低( $1.3 \%)$, 反映 了上海郊区居民的社区满意度总体较高(图4)。

\section{2 社区满意度的人群分异}

对不同社会经济属性、不同社区感知特征居民 的社区满意度进行比较, 并利用单因素方差分析 (one-way ANOVA)检验不同群体在社区满意度上是 否存在差异 ${ }^{\circledR}($ 表 2$)$ 。

方差分析结果显示:不同年龄、户口类别、婚姻 状况、家中小汽车拥有状况和住房产权状况的居民 在社区满意度上表现出显著差异, 其中不同户口类 别和产权状况的群体差异最为显著。男性的社区 满意度略低于女性, 但在统计上不显著。年龄越大 的居民,社区满意度越高, 尤其是 50 岁以上的居民, 满意度明显高于其他年龄段群体。有上海户口的 居民社区满意度远高于外地户口居民, 已婚居民社 区满意度也高于未婚居民。没有稳定工作的居民 大多是家庭主妇和退休老人, 具有较高的社区满意 度。家庭收人与社区满意度呈现倒“U”形的关系， 家庭平均月收人为 10000 20000元的居民社区满意 度最高。住房产权状况不同, 居民的社区满意度差 距十分明显, 拥有住房产权的居民社区满意度较 高。家庭拥有小汽车的居民社区满意度高于没有 小汽车的居民。

在社区感知特征方面, 对于社区的健康、便利、 安全、美观、交通拥堵情况具有不同评价水平的居 民, 他们的社区满意度也具有非常明显的差异, 反

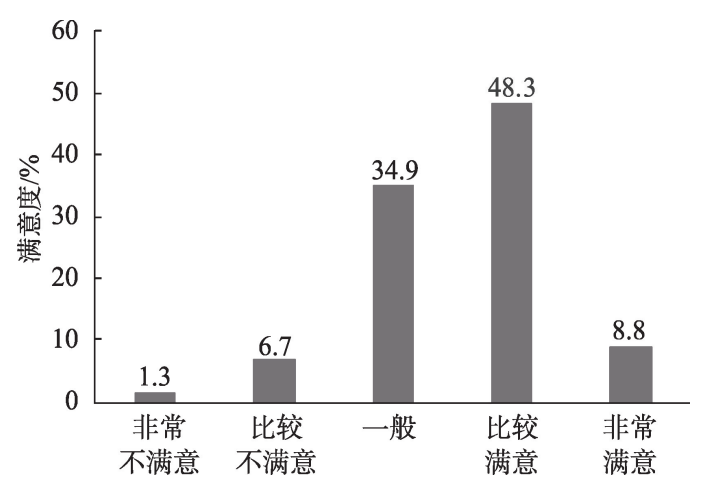

图 4 社区满意度分布

Fig.4 Residents' neighborhood satisfaction

映了社区感知特征与社区满意度之间的强相关性。

\section{4 社区满意度的影响因素}

\section{1 模型构建}

本文借鉴Campbell模型及 $\mathrm{CaO}(2015)$ 调整后的 Campbell模型,进行简化和调整后建立了本研究的 理论模型(图 5), 探讨个人与家庭社会经济属性、社 区客观特征、社区感知特征对社区满意度的影响。 将社会经济属性和社区客观特征作为外生变量, 社 区感知特征和社区满意度作为内生变量, 其中社区 感知特征为中介变量, 重点考察在外生变量的作用 下, 社区感知特征对社区满意度的影响。研究选择 结构方程模型方法开展分析, 利用 AMOS 22.0 软件 来实现。

\section{2 模型结果}

模型采用最大似然估计法拟合, 并用Bootstrap 估计法得到模型的方差-协方差矩阵。根据模型拟 合度的检验结果(表3), 几个重要的拟合度指标都达 到了理想水平, 模型拟合效果较好。

\subsection{1 社区感知特征对社区满意度的影响}

根据模型结果, 社区感知特征对社区满意度存 在显著的影响(表 4, 图6)。居民对社区总体健康、 总体便利、总体安全和总体美观的感知都对社区满 意度有显著的正影响,4种感知特征对社区满意度 的影响程度由高到低分别为健康、安全、美观、便 利, 对于郊区居民来说,这也可以看作是 4 种特征的 重要性排序。

首先, 社区总体健康的标准化回归系数Beta 最

(1) 方差分析采用社区满意度作为因变量,选取社会经济属性、社区感知特征两大类共 13 个指标作为自变量,用 SPSS 22.0 软件进行检验。 
表 2 居民的社区满意度特征比较

Tab.2 Result of one-way ANOVA of residents' neighborhood satisfaction

\begin{tabular}{|c|c|c|c|c|}
\hline \multirow{2}{*}{\multicolumn{2}{|c|}{ 变量 }} & \multirow{2}{*}{ 均值 } & \multicolumn{2}{|c|}{ 单因素方差分析 } \\
\hline & & & $F$ 值 & $P$ 值 \\
\hline \multicolumn{5}{|l|}{ 社会经济属性 } \\
\hline \multirow[t]{2}{*}{ 性别 } & 男性 & 3.54 & 1.109 & 0.351 \\
\hline & 女性 & 3.59 & & \\
\hline \multirow{4}{*}{ 年龄 } & 18 30岁 & 3.52 & 3.107 & $0.015^{* *}$ \\
\hline & $30 \sim 40$ 岁 & 3.54 & & \\
\hline & 40 50岁 & 3.55 & & \\
\hline & $50 \sim 60$ 岁 & 3.70 & & \\
\hline \multirow[t]{2}{*}{ 户口类别 } & 上海 & 3.64 & 4.161 & $0.002^{* * *}$ \\
\hline & 外地 & 3.48 & & \\
\hline \multirow[t]{2}{*}{ 婚姻状况 } & 已婚 & 3.59 & 1.980 & $0.095^{\circ}$ \\
\hline & 未婚 & 3.46 & & \\
\hline \multirow[t]{2}{*}{ 就业情况 } & 有稳定工作 & 3.55 & 1.867 & 0.114 \\
\hline & 无稳定工作 & 3.61 & & \\
\hline \multirow[t]{5}{*}{ 家庭平均月收人 } & 5000 元以下 & 3.50 & 0.403 & 0.807 \\
\hline & $5000 \sim 10000$ 元 & 3.56 & & \\
\hline & $10000 \sim 20000$ 元 & 3.61 & & \\
\hline & $20000 \sim 30000$ 元 & 3.52 & & \\
\hline & 30000 元以上 & 3.52 & & \\
\hline \multirow[t]{2}{*}{ 住房产权状况 } & 有住房产权 & 3.63 & 4.329 & $0.002^{2 * *}$ \\
\hline & 无住房产权 & 3.45 & & \\
\hline \multirow[t]{2}{*}{ 家庭是否拥有小汽车 } & 有小汽车 & 3.61 & 2.784 & $0.026^{\prime *}$ \\
\hline & 无小汽车 & 3.50 & & \\
\hline \multicolumn{5}{|l|}{ 社区感知特征 } \\
\hline 总体健康 & & & 72.344 & $<0.001^{\cdots * *}$ \\
\hline 总体便利 & & & 48.941 & $<0.001^{* * * *}$ \\
\hline 总体安全 & & & 70.542 & $<0.001^{* * *}$ \\
\hline 总体美观 & & & 64.358 & $<0.001^{* * *}$ \\
\hline 交通拥堵 & & & 4.082 & $0.003^{* * *}$ \\
\hline 总计 & & 3.57 & & \\
\hline
\end{tabular}

注: ***、***分别表示变量在 $0.1 、 0.05 、 0.01$ 的水平下显著。下同。

高 $(0.221)$, 反映了郊区居民对社区环境的总体健康 最为在意, 健康的社区环境最能提高居民的社区满 意度。研究表明, 安静、清洁的环境能够提高居民 的社区满意度(Hur et al, 2008; Lovejoy et al, 2010; Cao, 2015), 与市区相比, 郊区社区居住密度较低、 空气质量较好、环境相对安静, 是很多居民迁居郊 区的主要原因, 因此社区环境的健康程度对居民的 社区满意度影响最大。

其次为社区环境总体安全(标准化回归系数

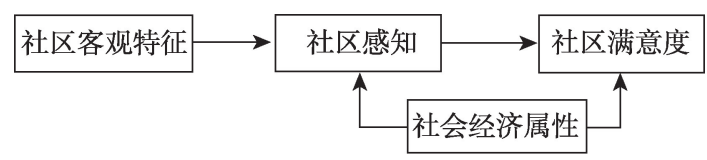

图 5 理论模型

Fig.5 The conceptual model
Beta 为 0.174) 和社区环境总体美观 (系数为 0.140 ), 这 2 种特征也对居民的社区满意度有较大的积极影 响。Wang D等(2016)对北京市居民社区满意度的 研究也发现, 居民对社区的安全性和外观的感知能 够显著提高居民的社区满意度, 与本文结果一致。

表 3 结构方程模型拟合情况

Tab.3 Goodness-of-fitting of the model

\begin{tabular}{llc}
\hline 拟合指数 & \multicolumn{1}{c}{ 参考值 } & 模型结果 \\
\hline CMIN/DF & $1 \sim 3$ & 2.620 \\
RMSEA & $<0.05$, 好; $<0.08$, 可接受 & 0.038 \\
SRMR & $<0.05$, 好; $<0.08$, 可接受 & 0.029 \\
CFI & $>0.90$ & 0.980 \\
\hline
\end{tabular}

注:卡方自由度比(CMIN/DF)、近似误差的均方根(RMSEA)和 标准化残差均方根 (SRMR) 为绝对拟合指数, 比较拟合指数 (CFI) 为 相对拟合指数。 
表 4 结构方程模型的标准化回归系数

Tab.4 Standardized coefficients of the structural equation model

\begin{tabular}{|c|c|c|c|c|c|c|c|c|c|c|c|c|}
\hline & \multicolumn{2}{|c|}{ 总体健康 } & \multicolumn{2}{|c|}{ 总体便利 } & \multicolumn{2}{|c|}{ 总体安全 } & \multicolumn{2}{|c|}{ 总体美观 } & \multicolumn{2}{|c|}{ 交通拥堵 } & \multicolumn{2}{|c|}{ 社区满意度 } \\
\hline & Beta & $P$ & Beta & $P$ & Beta & $P$ & Beta & $P$ & Beta & $P$ & Beta & $P$ \\
\hline \multicolumn{13}{|l|}{ 内生变量 } \\
\hline 总体健康 & NA & & NA & & NA & & NA & & NA & & 0.221 & $<0.001$ \\
\hline 总体便利 & NA & & NA & & NA & & NA & & NA & & 0.072 & 0.033 \\
\hline 总体安全 & NA & & NA & & NA & & NA & & NA & & 0.174 & $<0.001$ \\
\hline 总体美观 & NA & & NA & & NA & & NA & & NA & & 0.140 & $<0.001$ \\
\hline 交通拥堵 & NA & & NA & & NA & & NA & & NA & & & \\
\hline \multicolumn{13}{|l|}{ 外生变量 } \\
\hline \multicolumn{13}{|l|}{ 社区客观特征 } \\
\hline 职住距离 & & & & & & & & & -0.073 & 0.010 & NA & \\
\hline 人口密度 & -0.176 & $<0.001$ & -0.090 & 0.041 & -0.120 & 0.007 & -0.174 & $<0.001$ & 0.077 & 0.074 & NA & \\
\hline 地铁站可达性 & & & & & & & & & & & NA & \\
\hline 公交车站可达性 & 0.103 & 0.005 & 0.125 & 0.001 & 0.077 & 0.043 & & & 0.102 & 0.005 & NA & \\
\hline 公园广场可达性 & 0.127 & $<0.001$ & & & & & & & -0.096 & 0.004 & NA & \\
\hline 商业密度 & -0.099 & 0.028 & & & & & & & & & NA & \\
\hline 社区绿地率 & & & & & & & 0.051 & 0.100 & 0.115 & $<0.001$ & NA & \\
\hline 社区室外游乐设施 & 0.100 & 0.001 & 0.081 & 0.010 & 0.087 & 0.006 & 0.151 & $<0.001$ & -0.131 & $<0.001$ & NA & \\
\hline \multicolumn{13}{|l|}{ 社会经济属性 } \\
\hline 年龄 & 0.076 & 0.044 & & & & & & & & & & \\
\hline \multicolumn{13}{|l|}{ 男性 } \\
\hline 上海户口 & & & & & & & & & & & 0.063 & 0.045 \\
\hline \multicolumn{13}{|l|}{ 已婚 } \\
\hline \multicolumn{13}{|l|}{ 有稳定工作 } \\
\hline \multicolumn{13}{|l|}{ 家庭平均月收人 } \\
\hline \multicolumn{13}{|l|}{ 家庭有小汽车 } \\
\hline 拥有住房产权 & & & & & & & & & & & & \\
\hline
\end{tabular}

注:NA 表示变量之间没有直接联系; 无数据代表该变量的显著性低于 0.1 的水平。

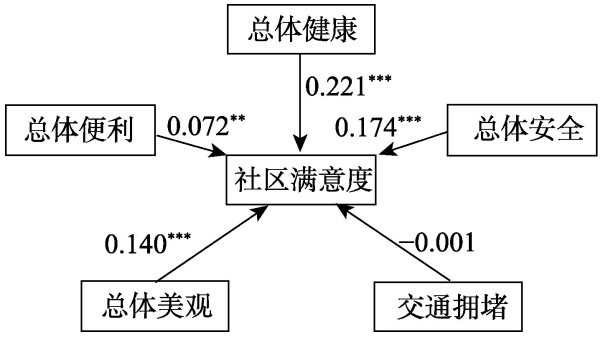

图 6 内生变量之间的关系

Fig.6 Relationship between the endogenous variables

在社区感知特征中, 影响最弱的是居民对社区 环境便利性的感知情况 (系数为 0.072 ), 即相对于健 康、安全、美观的社区环境而言, 是否便利对于社区 满意度也存在正向影响,但这种影响相对较弱。可 能存在 2 个方面原因:一方面尽管郊区社区的便利 情况相对较差, 但居民在此居住的主要目的是改善
居住和社区条件, 对于便利性情况的容忍度相对较 高; 另一方面已有研究发现较长的通勤距离形成的 时空制约会导致郊区居民在社区周边的购物和休 闲活动相对减少(申悦等, 2013; 刘志林等, 2015; 申 悦, 柴彦威, 2017), 因此居民对周边服务设施是否 便利的重视程度相对下降。

Gruber 等(1987)和 Hur 等(2008)的研究表明,交 通拥堵对居民的社区满意度存在显著影响,而在本 研究中居民对社区周边拥堵情况的感知对社区满 意度的影响不显著,这可能是由于郊区交通拥堵的 情况并不严重, 因此影响不大。

\subsection{2 社区客观特征的影响}

根据理论模型,社区客观特征直接影响居民对 社区的感知,从而通过社区感知特征间接地影响居 民的社区满意度。模型结果显示(表 4 ,表 5), 职住 
表 5 社区客观特征对社区满意度的标准化总影响

Tab.5 Standardized total effects of neighborhood characteristics on residents' neighborhood satisfaction

\begin{tabular}{lcc}
\hline \multicolumn{1}{c}{ 社区客观特征 } & 标准化的总影响 & $P$ \\
\hline 职住距离 & 0.004 & 0.541 \\
人口密度 & -0.091 & 0.010 \\
地铁站可达性 & -0.006 & 0.670 \\
公交车站可达性 & 0.050 & 0.010 \\
公园广场可达性 & 0.040 & 0.030 \\
商业密度 & -0.022 & 0.317 \\
社区绿地率 & 0.017 & 0.317 \\
社区室外游乐设施 & 0.064 & 0.016 \\
\hline
\end{tabular}

距离显著影响居民对社区周边交通拥堵的感知情 况, 职住距离越近的居民感知到的交通拥堵情况越 严重。这是由于职住距离近的居民在社区周边的 交通出行比例更高, 更容易受到社区周边交通状况 的影响,也更易感受到社区周边的交通拥堵情况。 但由于居民对社区周边交通拥堵情况的感知对社 区满意度没有显著影响, 因此职住距离对社区满意 度的总影响不显著。

人口密度对社区的总体健康、总体便利、总体 安全和总体美观 4 种特征都有显著的负影响, 同时 也会使居民感受到更严重的交通拥堵, 这与已有研 究结论一致(Cao et al, 2016)。人口密度的增加会使 社区的环境更加复杂, 噪音、污染等情况更严重, 交 通也更拥挤, 同时也会带来一定的治安问题。因 此, 社区的人口密度越高, 居民对社区环境及周边 交通特征都会有更糟糕的感受, 人口密度对社区满 意度的总影响消极且显著。

模型结果显示, 地铁站的可达性对社区感知特 征没有显著影响, 对社区满意度的总影响也不显 著, 而公交车站的可达性对总体健康、总体便利和 总体安全这 3 种感知特征却都有显著的正影响。这 可能是由于大多数郊区社区距离最近的地铁站都 超过了 $3 \mathrm{~km}$, 居民出行更多依赖公交车或私家车等 交通方式, 因此地铁站的可达性对居民日常出行的 影响较弱,也不会对社区满意度形成影响。同时, 由于现在的公交车系统越来越安全、高效和环保, 也能够促进步行等体力活动, 因此能够提高居民对 健康、便利和安全的感知。但是, 公交车站可达性 好也意味着社区周边的交通较为繁忙, 这就导致公 交车站的可达性越好, 居民感受到社区周边交通拥 堵的情况越严重。由于对交通拥堵程度的感知并 不影响社区满意度, 公交车站可达性对社区满意度
的总体影响是显著的积极影响。

公园广场的可达性显著影响居民对社区总体 健康和交通拥堵情况的感知。公园广场是居民休 闲娱乐和体育锻炼的重要场所, 植被多、绿化好, 这 些因素都容易使居民感受到生活在健康的环境 中。同时,由于公园和广场的开敞性能够疏散一定 的交通拥堵, 因此公园广场可达性越好, 居民更倾 向于认为社区周边交通拥堵不严重。公园广场可 达性对社区满意度有较为显著的积极总影响。

商业密度影响居民对社区总体健康的感知, 社 区周边商业密度越高, 居民感知到的社区环境的总 体健康情况越差。这可能是由于郊区的商业设施 大多规模较小、等级较低, 诸如餐馆之类的低端商 业设施可能对环境产生一定的负面效应, 因此会降 低居民对社区健康情况的评价。但另一方面,商业 密度的提高也能使居民的日常活动更加便利, 因此 商业密度对社区满意度的总影响并不显著。

社区绿地率能够提高居民对社区美观程度的 评价。同时,社区绿地率越高, 居民越能够感受到 社区周边交通拥堵,这可能是由于绿地率较高的社 区有更大概率是中高档社区, 居民出行对于小汽车 的依赖性更强, 对周边交通情况较为敏感; 也有可 能是社区绿地的增加导致了停车场地和机动车道 的相对减少, 容易造成停车难和社区周边的停车占 道问题。社区绿地率对社区满意度的总影响不 显著。

社区室外游乐设施对社区总体健康、总体便 利、总体安全和总体美观都有显著的正影响, 也能 降低居民对交通拥堵的感知。社区室外游乐设施 为居民提供家门口的室外活动场所, 不仅能让居民 在其中进行体育锻炼、亲子互动等活动, 也能够将 社区居民聚集起来,增进邻里关系。同时,此类场所 中有人的活动, 容易形成“街道眼”, 社区居民也就 更能感受到安全(简·雅各布斯, 2006)。因此, 社区 室外游乐设施对社区满意度的总影响是显著的正 影响。

\subsection{3 社会经济属性的影响}

模型结果显示,在综合考虑了社区客观特征、 社区感知特征对社区满意度的影响后,大多数社会 经济属性的影响变得不显著了, 只有年龄和户籍情 况对社区感知或社区满意度存在显著的影响。居 民对社区总体健康的感知受到年龄的显著影响,年 龄越大的居民, 认为社区环境总体上更健康, 这可 
能是由于年龄较大的居民更倾向于在社区内进行 体育锻炼等休闲活动, 更能感受到社区环境给自身 带来的健康。相比于外地户口居民, 有上海户口的 居民具有更高的社区满意度, 可能是由于上海户口 所带来的更多的社会福利、更好的社会资本和居住 条件, 这与已有研究的结论一致(湛东升等, 2014)。

\section{5 结论与讨论}

本文以居民的社区满意度为研究对象, 基于 Campbell模型构建理论模型, 从主客观环境相结合 的角度出发, 研究社区特征对社区满意度的影响机 理, 着重对其中社区主观感知的中介作用进行了探 讨, 并结合上海市郊区的特点开展实证研究, 利用 结构方程模型进行检验并分析其影响。研究结论 主要有:

(1) 居民的社区感知情况对于社区满意度具有 直接性和决定性的影响, 居民对社区总体健康、总 体便利、总体安全和总体美观的感知对社区满意度 都存在显著的正影响。

(2) 社区客观特征通过影响社区感知特征而间 接地影响社区满意度, 社区人口密度对社区满意度 有显著的消极影响, 公交车站可达性、公园广场可 达性和社区室外游乐设施对社区满意度的影响是 显著的正向影响, 商业密度对不同社区感知分别存 在正负影响, 它们之间相互抵消导致商业密度对社 区满意度的总影响不显著。

（3）居民的社会经济属性对社区感知特征和社 区满意度的影响甚微, 影响郊区居民社区满意度的 主要因素是社区的主客观特征。

本文研究结果对于上海市的郊区发展和社区 生活圈规划具有一定的借鉴意义。首先, 与西方城 市的郊区有所不同, 中国城市郊区的人口与建成环 境密度都相当高, 而较高的人口密度将对居民的社 区感知情况产生负面影响, 降低社区满意度, 因此 在郊区快速发展的过程中应对人口和建筑密度进 行一定的控制, 使其达到相对适中的水平; 其次, 社 区及周边的商业密度对于社区满意度的影响机理 较为复杂, 提升便利程度和降低健康感知程度的影 响同时存在, 因此郊区中的商业发展不应仅仅增加 设施数量, 更应该提高商业设施的等级和服务质 量, 实现 “质”与 “量” 的协调发展; 再次, 社区内的游 乐设施、小花园以及社区周边的公园、广场等开敞
空间都能够显著提高居民的社区满意度, 因此,在 郊区发展以及社区生活圈规划中应注重小规模开 敞空间的配置; 最后, 郊区居民家中拥有小汽车的 比例较高, 但公共交通可达性仍然对社区满意度有 较为显著的积极影响, 因此在郊区合理配置地铁和 公交站点将有利于引导居民使用更加健康、低碳的 出行方式,并提高他们的满意度。

本文研究也存在有待未来深人探讨的问题。 第一,将社区客观环境的界定范围设定为步行 15 $\min$ 可达的社区周边 $1 \mathrm{~km}$ 范围内, 但郊区的特点导 致居民的出行方式可能不完全以步行为主, 其日常 活动半径也不仅限于 $1 \mathrm{~km}$ 范围内, 因此郊区社区生 活圈的空间尺度以及居民的日常活动空间范围有 待进一步探讨; 第二, 研究发现诸如商业密度之类 的外生变量可能对不同的感知特征同时存在正负 影响, 从而导致对社区满意度的总影响不显著, 在 本文采用的模型中,这种复杂的影响机制难以得到 明确解释, 在未来研究中有待深人挖掘; 第三, 研究 参考 Campbell 模型, 主要将居民对社区的主观感知 和社区满意度作为内生变量加以重点考虑, 但实际 上这二者与居民在社区内的日常活动也息息相关， 因此社区感知、社区活动和社区满意度之间的相互 作用机理有待未来深人研究。

\section{参考文献(References)}

柴彦威, 申悦, 陈梓烽. 2014. 基于时空间行为的人本导向的

智慧城市规划与管理 [J]. 国际城市规划, 29(6): 31-37, 50. [Chai Y W, Shen Y, Chen Z F. 2014. Towards smarter cities: Human- oriented urban planning and management based on space-time behavior research. Urban Planning International, 29(6): 31-37, 50. ]

陈卉, 甄峰. 2016. 信息通讯技术对老年人的社区满意度影 响路径: 以南京市锁金社区为例 [J]. 地理科学进展, 35 (9): 1167-1176. [Chen H, Zhen F. 2016. Impact of ICT on community satisfaction among elderly: The case of Suojin Community in Nanjing City. Progress in Geography, 35 (9): 1167-1176. ]

党云晓, 余建辉, 张文忠, 等. 2016. 环渤海地区城市居住环 境满意度评价及影响因素分析 [J]. 地理科学进展, 35 (2): 184-194. [Dang Y X, Yu J H, Zhang W Z, et al. 2016. Satisfaction evaluation of living environment and influencing factors in the Bohai Rim area. Progress in Geography, 35(2): 184-194. ]

段雪辉. 2016. 大型居住社区居民社区满意度研究 [J]. 城市 观察, (1): 85-95. [Duan X H. 2016. Research on communi- 
ty satisfaction of residents in large residential communities. Urban Insight, (1): 85-95. ]

冯健, 林文盛. 2017. 苏州老城区衰退邻里居住满意度及影 响因素 [J]. 地理科学进展, 36(2): 159-170. [Feng J, Lin W S. 2017. Residential satisfaction level and influencing factors of declining old town residents in Suzhou. Progress in Geography, 36(2): 159-170. ]

顾朝林. 2017. 基于地方分权的城市治理模式研究: 以新城 新区为例 [J]. 城市发展研究, 24(2): 70-78. [Gu C L. 2017. Research on urban governance models based on decentralization: Focus on new towns and new development areas. Urban Development Studies, 24(2): 70-78. ]

何深静, 齐晓玲. 2014. 广州市三类社区居住满意度与迁居 意愿研究 [J]. 地理科学, 34(11): 1327-1336. [He S J, Qi X L. 2014. Determinantd of relocation satisfaction and relocation intention in Chinese Cities: An empirical investigation on three types of residential neighborhood in Guangzhou. Scientia Geographica Sinica, 34(11): 1327-1336. ]

简 - 雅各布斯. 2006. 美国大城市的死与生 [M]. 南京: 译林出 版社: 25-47. [Jacobs J. 2006. The death and life of great american cities. Nanjing, China: Yilin Press: 25-47. ]

李俊峰, 高凌宇, 马作幸. 2017. 跨江择居居民的居住满意度 及影响因素: 以南京市浦口区为例 [J]. 地理研究, 36 (12): 2383-2392. [Li J F, Gao L Y, Ma Z X. 2017. Residential satisfaction and its influencing factors of the residents choosing to live trans- Yangtze River. Geographical Research, 36(12): 2383-2392. ]

刘志林, 廖露, 钮晨琳. 2015. 社区社会资本对居住满意度的 影响: 基于北京市中低收人社区调查的实证分析 [J]. 人 文地理, 30(3): 21-27, 71. [Liu Z L, Liao L, Niu C L. 2015. Residential satisfaction of community social capital: an empirical study of middle and low income residents in urban Beijing. Human Geography, 30(3): 21-27, 71. ]

宁越敏. 2012. 中国城市化特点、问题及治理 [J]. 南京社会科 学, (10): 19-27. [Ning Y M. 2012. A study on the urbanization, urban problems and solution in China. Social Sciences in Nanjing, (10): 19-27. ]

仇保兴. 2012. 新型城镇化: 从概念到行动 [J]. 行政管理改 革, (11): 11-18. [Qiu B X. 2012. New urbanization: From conception to action. Administration Reform, (11): 11-18. ] 单菁菁. 2008. 社区归属感与社区满意度 [J]. 城市问题, (3): 58-64. [Shan J J. 2008. Community attachment and community satisfaction. Urban Problems, (3):58-64.]

上海市人民政府. 2016. 上海市城市总体规划 2017-2035

[Z]. 上 海. [Shanghai Municipal Peoples Government. 2016. Shanghai master plan 2017-2035. Shanghai, China. ] 申悦, 柴彦威. 2013. 基于 GPS 数据的北京市郊区巨型社区
居民日常活动空间 [J]. 地理学报, 68(4): 506-516. [Shen Y, Chai Y W. 2013. Daily activity space of suburban mega-community residents in Beijing based on GPS data. Acta Geographica Sinica, 68(4): 506-516. ]

申悦, 柴彦威. 2017. 基于性别比较的北京城市居民活动的 时空弹性研究 [J]. 地理学报, 72(12): 2214-2225. [Shen Y, Chai Y W. 2017. Space-time flexibility of daily activities and gender differences: A case study of Beijing. Acta Geographica Sinica, 72(12): 2214-2225. ]

申悦, 柴彦威. 2018. 基于日常活动空间的社会空间分异研 究进展 [J]. 地理科学进展, 37(6): 853-862. [Shen Y, Chai Y W. 2018. Progress of research on sociospatial differentiation based on daily activity space of urban residents. Progress in Geography, 37(6): 853-862. ]

申悦, 塔娜, 柴彦威. 2017. 基于生活空间与活动空间视角的 郊区空间研究框架 [J]. 人文地理, 32(4): 1-6. [Shen Y, Ta N, Chai Y W. 2017. Research framework of suburban space based on perspective of living space and activity space. Human Geography, 32(4): 1-6. ]

王丰龙, 王冬根. 2015. 主观幸福感度量研究进展及其对智 慧城市建设的启示 [J]. 地理科学进展, 34(4): 482-493.

[Wang F L, Wang D G. 2015. Measures of subjective well-being: A review. Progress in Geography, 34(4): 482493. ]

王娟. 2016. 城中村改造安置区村民居住满意度调查: 以郑 州城中村改造为例 [J]. 建筑学报, 14(S1): 86-89. [Wang J. 2016. A study of living satisfaction in the urban village relocation areas: The case of Zhengzhou urban villages. Architectural Journal, 14(S1): 86-89. ]

魏立华, 间小培. 2006. 大城市郊区化中社会空间的“非均衡 破碎化”: 以广州市为例 [J]. 城市规划, 30(5): 55-60, 87. [Wei L H, Yan X P. 2006. Unbalanced and segmented urban social space in suburbanization: A case of metropolis Guangzhou of China. City Planning Review, 30(5): 55$60,87$.

吴缚龙, 沈洁. 2015. 中国城市的郊区开发和治理 [J]. 国际城 市规划, 30(6): 27-33. [Wu F L, Shen J. 2015. Suburban development and governance in China. Urban Planning International, 30(6): 27-33. ]

奚东帆, 吴秋晴, 张敏清, 等. 2017. 面向 2040 年的上海社区 生活圈规划与建设路径探索 [J]. 上海城市规划, (4): 6569. [Xi D F, Wu Q Q, Zhang M Q, et al. 2017. Exploration of planning and construction of community living circle in Shanghai facing 2040. Shanghai Urban Planning Review, (4): 65-69. ]

夏永久, 朱喜钢. 2013. 城市被动式动迁居民社区满意度评 价研究: 以南京为例 [J]. 地理科学, 33(8): 918-925. [Xia Y J, Zhu X G. 2013. The evaluation of urban forced mov- 
ers' community satisfaction: A case study of Nanjing. Scientia Geographica Sinica, 33(8): 918-925. ]

许丹纯, 俞守华, 区晶莹. 2014. 新市民社区生活满意度调查 [J]. 城市问题, (10): 96-100. [Xu D C, Yu S H, Qu J Y. 2014. Research of new citizens life satisfaction in new communities. Urban Problems, (10): 96-100. ]

姚士谋, 张平宇, 余成, 等. 2014. 中国新型城镇化理论与实 践问题 [J]. 地理科学, 34(6): 641-647. [Yao S M, Zhang P Y, Yu C, et al. 2014. The theory and practice of new urbanization in China. Scientia Geographica Sinica, 34(6): 641-647. ]

袁媛, 丁凯丽, 曹新宇, 等. 2018. 社区满意度及影响因素研 究方法综述 [J]. 城市发展研究, 25(10): 105-111. [Yuan Y, Ding K L, Cao X Y, et al. 2018. A review of neighborhood satisfaction. Urban Development Studies, 25(10): 105-111. ]

湛东升, 孟斌, 张文忠. 2014. 北京市居民居住满意度感知与 行为意向研究 [J]. 地理研究, 33(2): 336-348. [Zhan D S, Meng B, Zhang W Z. 2014. A study on residential satisfaction and its behavioral intention in Beijing. Geographical Research, 33(2): 336-348. ]

张景秋, 刘欢, 齐英茜, 等. 2015. 北京城市老年人居住环境及 生活满意度分析 [J]. 地理科学进展, 34(12): 1628-1636 [Zhang J Q, Liu H, Qi Y Q, et al. 2015. Living environment and life satisfaction of aged population in Beijing Municipality. Progress in Geography, 34(12): 16281636. ]

赵东霞, 卢小君. 2012. 城市社区居民满意度评价研究: 以高 档商品房社区和旧居住社区为例 $[\mathrm{J}]$. 大连理工大学学 报(社会科学版), 33(2): 93-98. [Zhao D X, Lu X J. 2012 Evaluation of urban community residents' satisfaction based on AHP: With high-grade commercial house community and old residential community as examples. Journal of Dalian University of Technology (Social Sciences), 33(2): 93-98. ]

邹晖, 罗小龙, 涂静宇. 2014. 基于小产权房居住满意度的实 证研究: 以南京迈臬桥地区小产权房社区为例 $[\mathrm{J}]$. 人文 地理, 29(1): 61-65. [Zou H, Luo X L, Tu J Y. 2014. An empirical study on living satisfaction of the restricted property house: A case study of Maigaoqiao in Nanjing. Human Geography, 29(1): 61-65. ]

Adams R E. 1992. Is happiness a home in the suburbs? The influence of urban versus suburban neighborhoods on psychological health $[\mathrm{J}]$. Journal of Community Psychology, 20(4): 353-372.

Ansari P. 2002. What makes people dissatisfied with their
Neighbourhoods? [J]. Urban Studies, 39(13): 2413-2438.

Baba Y, Austin D M. 1989. Neighborhood environmental satisfaction, victimization, and social participation as determinants of perceived neighborhood safety [J]. Environment \& Behavior, 21(6): 763-780.

Campbell A, Converse P, Rodgers W. 1976. The quality of american life: Perceptions, evaluations and satisfactions [M]. New York, NY: Russell Sage Foundation.

Cao X. 2015. How does neighborhood design affect life satisfaction? Evidence from Twin Cities [J]. Travel Behavior \& Society, 5: 68-76.

Cao X, Wang D. 2016. Environmental correlates of residential satisfaction: An exploration of mismatched neighborhood characteristics in the Twin Cities [J]. Landscape \& Urban Planning, 150: 26-35.

Cook C C. 1988. Components of neighborhood satisfaction responses from urban and suburban single-parent women [J]. Environment \& Behavior, 20(2): 115-149.

Gruber K J, Shelton G G. 1987. Assessment of neighborhood satisfaction by residents of three housing types [J]. Social Indicators Research, 19(3): 303-315.

Hur M, Morrow- Jones H. 2008. Factors that influence residents' satisfaction with neighborhoods [J]. Environment \& Behavior, 40(5): 619-635

Lee B A, Guest A M. 1983. Determinants of neighborhood satisfaction: A metropolitan-level analysis [J]. Sociological Quarterly, 24(2): 287-303.

Lovejoy K, Handy S, Mokhtarian P. 2010. Neighborhood satisfaction in suburban versus traditional environments: An evaluation of contributing characteristics in eight California neighborhoods [J]. Landscape \& Urban Planning, 97 (1): 37-48.

Lu M. 1999. Determinants of residential satisfaction: Ordered logit vs. regression models [J]. Growth \& Change, 30(2): 264-287.

Permentier M, Bolt G, Ham M V. 2011. Determinants of neighbourhood satisfaction and perception of neighbourhood reputation [J]. Urban Studies, 48(5): 977-996.

Wang D, Wang F. 2016. Contributions of the usage and affective experience of the residential environment to residential satisfaction [J]. Housing Studies, 31(1): 1-19.

Wang F, Wang D. 2016. Geography of urban life satisfaction: An empirical study of Beijing [J]. Travel Behaviour \& Society, 5: 14-22.

Zhou Y, Logan J R. 2008. Growth on the edge: The new Chinese metropolis [M]. Oxford, UK: Blackwell: 140-160. 


\title{
Impact of the built environment and perceived neighborhood characteristics on residents' satisfaction : Evidence from the Shanghai suburbs
}

\author{
SHEN Yue ${ }^{1,2}$, FU Xingxing \\ (1. The Center for Modern Chinese City Studies, East China Normal University, Shanghai 200062, China; \\ 2. School of Urban and Regional Science, East China Normal University, Shanghai 200062, China; \\ 3. School of Architecture and Urban Planning, Nanjing University, Nanjing 210093, China)
}

\begin{abstract}
With human-centered development as the key principle of urban development in China, urban studies and urban planning are paying more attention to the construction of urban living space and the improvement of residents' life quality, and the importance of research on residents' neighborhood satisfaction is increasing. Under the background of rapid suburbanization and the construction of community life circle, this study used primary data on residents' daily activities and travel behaviors from 10 towns in the Shanghai suburbs to empirically investigate residents' neighborhood satisfaction. We constructed a theoretical model based on Campbell's Model, and then examined the interactions between neighborhood built environment, perceived neighborhood characteristics, and residents' neighborhood satisfaction by combining subjective and objective assessments. A structural equation model was used to verify the impact pathways and mechanisms. The results show that perceived neighborhood characteristics have direct and decisive influences on residents' neighborhood satisfaction, and residents' perceptions on the neighborhood situation of health, convenience, safety, and appearance all have significant positive impacts on residents' satisfaction. Factors of the built environment such as density, accessibility, and facility situation impact residents' satisfaction indirectly through perceived neighborhood characteristics. Residents' socioeconomic characteristics have little influence on their neighborhood satisfaction, and the main influencing factors of residents' satisfaction are neighborhood characteristics.
\end{abstract}

Keywords: built environment; perceived neighborhood characteristics; community life circle; structural equation model; suburbs; Shanghai 